
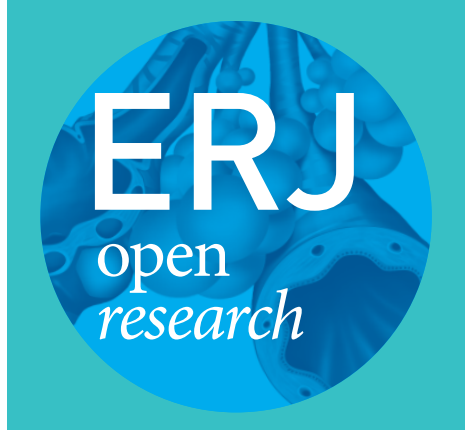

\section{An algorithmic approach for the treatment of severe uncontrolled asthma}

\author{
Eleftherios Zervas ${ }^{1}$, Konstantinos Samitas ${ }^{1}$, Andriana I. Papaioannou², \\ Petros Bakakos ${ }^{3}$, Stelios Loukides ${ }^{2}$ and Mina Gaga ${ }^{1}$
}

Affiliations: ${ }^{17 t h ~ R e s p i r a t o r y ~ M e d i c i n e ~ D e p t ~ a n d ~ A s t h m a ~ C e n t e r, ~ A t h e n s ~ C h e s t ~ H o s p i t a l ~ " S o t i r i a ", ~ A t h e n s, ~}$ Greece. ${ }^{2} 2$ nd Respiratory Medicine Dept, Attikon Hospital, University of Athens, Athens, Greece. ${ }^{3} 1$ st Respiratory Medicine Dept, Athens Chest Hospital "Sotiria”, University of Athens, Athens, Greece.

Correspondence: Eleftherios Zervas, 7th Respiratory Medicine Dept and Asthma Center, Athens Chest Hospital "Sotiria", 152 Mesogeion Avenue, Athens 115 27, Greece. E-mail: lefzervasdyahoo.gr

ABSTRACT A small subgroup of patients with asthma suffers from severe disease that is either partially controlled or uncontrolled despite intensive, guideline-based treatment. These patients have significantly impaired quality of life and although they constitute $<5 \%$ of all asthma patients, they are responsible for more than half of asthma-related healthcare costs. Here, we review a definition for severe asthma and present all therapeutic options currently available for these severe asthma patients. Moreover, we suggest a specific algorithmic treatment approach for the management of severe, difficult-to-treat asthma based on specific phenotype characteristics and biomarkers. The diagnosis and management of severe asthma requires specialised experience, time and effort to comprehend the needs and expectations of each individual patient and incorporate those as well as his/her specific phenotype characteristics into the management planning. Although some new treatment options are currently available for these patients, there is still a need for further research into severe asthma and yet more treatment options.

@ERSpublications

Stepwise approach for the treatment of severe asthma http://ow.ly/rLPl30i0TyZ

Cite this article as: Zervas E, Samitas K, Papaioannou AI, et al. An algorithmic approach for the treatment of severe uncontrolled asthma. ERJ Open Res 2018; 4: 00125-2017 [https://doi.org/10.1183/ 23120541.00125-2017].

Received: Oct 102017 | Accepted after revision: Jan 142018

Conflict of interest: None declared.

Copyright $\odot$ ERS 2018. This article is open access and distributed under the terms of the Creative Commons Attribution Non-Commercial Licence 4.0. 


\section{Introduction}

Asthma is a common, chronic and heterogeneous disease, affecting people of all ages. It may be mild, barely noticed by the patient, or it may range all the way to very severe disease, causing constant symptoms greatly affecting the life of the patient, and may result in poor quality of life and severe, life-threatening attacks. Severe asthma is increasingly associated with different specific phenotypes and it represents a major unmet therapeutic need [1]. Approximately $5-10 \%$ of patients are estimated to suffer from severe asthma, whereas $1-2 \%$ have severe treatment-resistant/refractory asthma, but there is significant geographic variation [2].

During the last few years, novel research and clinical studies have provided new information regarding severe asthma phenotyping, endotyping and treatment options. We have come to realise that the identification of the patient's specific inflammatory phenotype is important not only for research purposes, but also to implement a successful treatment plan. Early studies involved mainly allergic asthma and early/ late allergic reactions, which can be easily reproduced in experimental models, and accordingly it has been widely accepted that asthma is primarily a T-helper (Th) 2 immunological disease. However, recent studies on severe asthma have shown that in many cases it is nonatopic, and that inflammation in severe asthma is not characterised by eosinophilia and Th2-type cytokines, but may in fact be T2-low. Severe asthma cohort studies (e.g. the ENFUMOSA/BIOAIR studies [3-5], the TENOR/SARP studies [6-8], the UK and Belgium severe asthma registries [9, 10], and, more recently, the U-BIOPRED project [11]) established the value of phenotyping severe asthma and show that some patients have stable eosinophilic phenotypes over time, while others are steadily noneosinophilic or alternate between phenotypes.

This information has led to the development of new treatment options in severe asthma, such as the use of monoclonal antibodies (mAbs) or specific pathway inhibitors that have demonstrated clinical efficacy in very well-designed clinical studies [12-15]. These therapeutic agents have been formulated to target specific pheno- and/or endotypes. Therefore, in everyday clinical practice treating physicians now have several therapeutic options for severe asthma, but also have the difficult task of identifying which patient is the best candidate for each of these specific treatments. In this review, we will summarise currently available therapeutic options for severe asthma and propose a pheno-endotype-based treatment algorithmic approach in patients with severe uncontrolled asthma.

\section{Severe asthma definition}

The definition of asthma severity has changed considerably over the years. In the early Global Initiative for Asthma (GINA) and National Asthma Education and Prevention Program (NAEPP) guidelines, overall asthma severity was considered mainly on the basis of the patient's clinical characteristics prior to initiation of treatment $[16,17]$. Recent GINA/NAEPP guidelines define asthma severity based on the level of asthma treatment used to maintain adequate asthma control $[18,19]$. Asthma that requires continuous high-dose treatment has important medication side-effects and high long-term risk. This severe form of asthma, often referred to as treatment-resistant, refractory or difficult-to-treat asthma, has been the subject of a number of reports published by the European Respiratory Society (ERS) and American Thoracic Society (ATS) [2, 11, 20-23]. According to the current ERS/ATS Task Force on severe asthma, the proposed definition describes severe asthma as asthma which requires treatment with high-dose inhaled corticosteroids (ICSs) plus a second controller (and/or systemic corticosteroids) to prevent it from becoming uncontrolled or which remains uncontrolled despite this therapy [2]. This definition therefore includes treatment-resistant asthma and severe asthma that persists due to inability to effectively treat confounders/comorbidities, such as severe sinus disease or obesity, but does not include untreated asthma.

Therefore, the vast majority of these patients will have already received high doses of ICSs plus long-acting $\beta$-agonists (LABAs) as a second controller and in most cases in fixed combinations. A significant proportion of these patients are already on add-on treatment with tiotropium and/or leukotriene receptor antagonists (LTRAs) and/or theophylline. The definition of high-dose ICS proposed by the ERS/ATS is higher that that used in clinical practice (table 1). Fixed combinations do not allow for ICS doses to reach these high levels without exceeding safety levels for their LABA component. Adding ICS in separate inhalers has not been tested in clinical studies and is generally not advised by severe asthma experts worldwide. In our opinion, GINA-based ICS dose ranges seem closer to real-life settings (table 1).

\section{Severe asthma phenotypes}

Characterising asthma patients based on their corresponding endotypes allows better phenotyping and guidance of available treatment options. Currently, two major endotypes of severe asthma have been identified: T2-high and T2-low asthma. T2-high asthma is associated with increased epithelial expression of Th2-type cytokines, such as interleukin (IL)-4, IL-5 and IL-13. Although heterogeneous in nature, the designation of the T2-high endotype has been primarily based on the presence of atopy and/or 
TABLE 1 High-dose inhaled corticosteroids proposed by the European Respiratory Society (ERS)/American Thoracic Society (ATS) [2] and the Global Initiative for Asthma (GINA) [18]

\begin{tabular}{lcc} 
& $\begin{array}{c}\text { ERS/ATS } \\
\text { high dose } \boldsymbol{\mu g}\end{array}$ & $\begin{array}{c}\text { GINA } \\
\text { high dose } \boldsymbol{\mu g}\end{array}$ \\
\hline Beclomethasone dipropionate (chlorofluorocarbon) & $\geqslant 2000$ & $>1000$ \\
Beclomethasone dipropionate (hydrofluoroalkane) & $\geqslant 1000$ & $>400$ \\
Budesonide & $\geqslant 1600$ & $>800$ \\
Ciclesonide & $\geqslant 320$ & $>320$ \\
Fluticasone furoate & $N A$ & 200 \\
Fluticasone propionate & $\geqslant 1000$ & $>500$ \\
Mometasone furoate & $\geqslant 800$ & $\geqslant 440$ \\
Triamcinolone acetonide & $\geqslant 1200$ & $>2000$ \\
\hline NA: not applicable. & & \\
\hline
\end{tabular}

eosinophilic airway inflammation, usually identified on the basis of sputum or blood eosinophilia, yet with no universally accepted thresholds $[13,15,24-26]$. The definition of the T2-low endotype is even more elusive. It is usually defined by the absence of markers of Th2-mediated inflammation and is characterised by neutrophilic or, less commonly, paucigranulocytic airway infiltration depicted in induced sputum cell counts [27]. Other aspects of severe asthma pathophysiology, such as recurrent bacterial/viral infections, altered innate immunity responses and airway remodelling, may also contribute mostly to T2-low asthma.

In clinical practice, surrogate markers are essential in phenotyping severe asthma patients and in guiding treatment. Total and/or specific IgE levels as well as skin prick test (SPT) positivity have long been used as markers of atopy. Moreover, as analysis of induced sputum is usually performed in highly specialised centres, several other T2-high asthma biomarkers have been thoroughly examined and validated up to now, such as exhaled nitric oxide (NO), blood eosinophils and serum periostin [28]. However, biomarkers for T2-low asthma are still lacking. Therefore, characterising the T2-low endotype essentially requires the lack of any known biomarkers of T2-high asthma. In a recent study by Busse et al. [29], cut-off points used to define high levels of Th2 immune activation were described (IgE $\geqslant 100 \mathrm{IU} \cdot \mathrm{mL}^{-1}$, blood eosinophil count $\geqslant 300 \mu \mathrm{L}^{-1}$ and exhaled NO fraction ( FENO) $\geqslant 30 \mathrm{ppb}$ ). Using these thresholds, patients can be classified as having either a high (elevation in two or more T2 biomarkers) or a low Th2 (elevation in one or no T2 biomarkers) immune profile [29]. This approach seems reasonable until we find a T2-low biomarker validated in well-designed studies that can be used in clinical practice.

\section{Treating severe asthma: current options}

During the last decade several new drugs addressing severe asthma have been developed and approved or are in phase 3 clinical studies. Some of these drugs, such as omalizumab or anti-IL-5 mAbs, have recently been included in Step 5 in the latest GINA recommendations [18], along with other established treatment options, such as tiotropium and oral corticosteroids (OCSs).

\section{Tiotropium}

Tiotropium is a long-acting muscarinic antagonist (LAMA) with duration of action exceeding $24 \mathrm{~h}$, attributed to its slow dissociation from muscarinic receptors [30]. In animal models of allergic asthma, it has been shown that tiotropium has anti-inflammatory effects [31, 32]. The mechanisms by which LAMAs have the potential to improve asthma control include bronchodilation or inhibition of cholinergically mediated bronchoconstriction, but also possible inhibition of airway smooth muscle hypertrophy and hyperresponsiveness [33].

In a proof-of-concept study, including patients on GINA Step 4 treatment with severe persistent asthma, tiotropium add-on treatment provided highly significant improvements in peak forced expiratory volume in $1 \mathrm{~s}$ (FEV1) [34]. KeRSTJENs et al. [35] reported results from two long-term, replicate, phase 3 trials of tiotropium Respimat $5 \mu \mathrm{g}$, also in patients with symptomatic severe asthma. In this cohort of 912 patients with $\mathrm{FEV}_{1}<80 \%$ pred, tiotropium Respimat significantly increased the time to the first severe exacerbation and improved lung function. However, improvements in Asthma Control Questionnaire (ACQ)-7 and Asthma Quality of Life Questionnaire (AQLQ) scores were small and inconsistent, and did not reach a clinically important difference. Similar results were reported in a real-life asthma population, where the addition of tiotropium was associated with significant decreases in the incidence of exacerbations (from $37 \%$ to $27 \%$ ) and antibiotic prescriptions for lower respiratory tract infections [36]. Tiotropium (soft-mist 
inhaler) has recently been included as a new add-on treatment for GINA Steps 4 and 5 in patients aged $\geqslant 18$ years with a history of exacerbations (Evidence A) [18].

\section{Anti-IgE treatment (omalizumab)}

IgE is an important mediator of allergic reactions and it was considered early as a suitable therapeutic target [37]. Omalizumab is the first recombinant humanised IgG1 mAb, which targets and binds free IgE, interrupting the IgE-mediated asthma inflammatory cascade at an early stage, and thus reducing both early and late asthmatic responses [38]. Patients in which omalizumab can be considered as an add-on therapy are patients with severe asthma and at least one positive SPT or serum IgE to a perennial aeroallergen (radioallergosorbent test (RAST)), reduced lung function (FEV1 $<80 \%$ pred) and multiple documented exacerbations of asthma despite receiving daily high-dose ICS plus a LABA. In these patients, randomised controlled clinical trials have shown that omalizumab significantly decreases asthma exacerbations, including hospital admission and emergency room visits, improves respiratory symptoms and quality of life, and reduces the use of OCSs, ICSs and rescue medication [12, 39-41]. These favourable outcomes of clinical trials have been confirmed and enhanced by multiple real-life studies, also providing evidence for the long-term efficacy of this drug [42-45]. Furthermore, despite initial concerns, omalizumab has positive short- and long-term safety profiles in both randomised clinical trials and real-life studies $[45,46]$. Of note, in the latest GINA update, omalizumab has replaced oral steroids as the preferred add-on option in severe allergic asthma [18].

Not all patients with severe allergic asthma and high IgE levels will benefit from omalizumab. Patients should be reassessed 16 weeks after treatment initiation in order to evaluate their response to treatment and to decide whether the treatment should be continued [47]. Baseline characteristics do not reliably predict response to omalizumab, but studies have shown that patients more likely to benefit might be those with high levels of Th2 inflammatory biomarkers, such as exhaled NO, blood eosinophils and serum periostin [48]. The optimal duration of omalizumab treatment has not yet been determined. Due to the mechanism of action of omalizumab, once administered, it should probably be continued. A relapse in asthma symptoms and exacerbations within a few months after anti-IgE discontinuation has been previously reported [49]. In a recently published placebo-controlled study, the risks and benefits of continuing or not omalizumab after long-term use ( $\sim 5$ years $)$ was assessed. The results demonstrate that continuation of omalizumab resulted in sustained benefit in terms of symptoms control and exacerbations [50]. Nevertheless, the relatively high percentage of subjects in the placebo arm with no exacerbations and good asthma control did suggest a persistency of benefit even if the long-term omalizumab treatment is discontinued [50]. Adverse events are rare and mostly represent local reactions at injection sites. Headache, nausea or tiredness have been also reported, but the frequency does not differ from that of placebo. Anaphylactic reactions have been occasionally reported, but are extremely rare.

\section{Anti-IL-5 treatment}

IL-5 is the only cytokine activating the high-affinity IL-5 receptor expressed primarily in eosinophils and basophils. IL-5 promotes the differentiation, expansion and survival of eosinophils. IL-5 is also responsible for eosinophil activation and degranulation [51]. As eosinophils are pivotal in allergic reactions, the central role that IL-5 plays in eosinophil biology makes it an ideal target for attenuating these responses. Severe asthma has for long been associated with increased activity of circulating and tissue eosinophils [52]. To date, two humanised mAbs, mepolizumab and reslizumab, that bind to human IL-5 have been developed. These antibodies have already been tested in animal models as well as in several studies involving patients primarily with severe asthma [13, 15, 24, 53-57]. In addition, a mAb (benralizumab) targeting the IL-5 receptor has been recently developed and studied in uncontrolled eosinophilic asthma [58-60].

\section{Mepolizumab}

Mepolizumab is a mouse anti-human mAb which is specific for human IL-5. It acts by binding to human IL-5 with high affinity and specificity; thus, it effectively blocks IL-5 from binding to the IL-5 receptor complex on the cell surface of eosinophils [61]. Mepolizumab is currently in clinical trials in a broad spectrum of eosinophilic-derived diseases, such as hypereosinophilic syndrome, eosinophilic oesophagitis, eosinophilic granulomatosis with polyangiitis, nasal polyposis as well as in severe asthma and chronic obstructive pulmonary disease (COPD).

Initial studies with mepolizumab in mild to moderate asthma were negative in terms of clinical benefit, despite a significant reduction in blood and sputum eosinophils [62, 63]. These frustrating results led several investigators to question the efficacy of this targeted therapy. However, following a more careful selection of the study population, based on the dogma that targeted therapy is for specific phenotypes/ endotypes of a disease, the perspective started to change. Two small but significant studies published in 2009 investigated the role of mepolizumab in severe uncontrolled asthma with persistent eosinophilia 
(sputum eosinophils $>3 \%$ ) $[13,24]$. In terms of clinical efficacy, treatment with mepolizumab was associated with significantly fewer severe exacerbations than placebo and a significant reduction in oral prednisone dose $[13,24]$. However, no significant differences were observed with respect to symptoms, FEV1 or airway hyperresponsivness $[13,24]$. The results of these two studies indicate that anti-IL-5 treatment can be effective in a carefully selected subgroup of asthmatic patients with persistent eosinophilia, in whom eosinophils seem to be important contributors to the pathophysiology of asthma exacerbations.

In 2012, the results of a large multicentre, placebo-controlled trial (the DREAM study), which recruited 621 patients with a history of recurrent severe asthma exacerbations and signs of eosinophilic inflammation, was published [15]. Mepolizumab significantly reduced the number of asthma exacerbations, but, similar to previous studies, had a small effect on traditional markers of asthma control, i.e. FEV1, AQLQ and ACQ scores [15]. In this study, only baseline peripheral blood eosinophil count and exacerbation frequency in the previous year were associated with efficacy [15]. Based on this finding, two studies in severe eosinophilic asthma defined by blood eosinophils levels were published in 2014 [55, 56]. In the MENSA study, mepolizumab administered either intravenously or subcutaneously significantly reduced asthma exacerbations (32\% and 61\%, respectively) and improved markers of asthma control [55]. BEL et al. [56] studied mepolizumab therapy in 135 patients with severe eosinophilic corticosteroid-dependent asthma. In these patients requiring daily oral glucocorticoid therapy, mepolizumab had a significant glucocorticoid-sparing effect (50\% reduction in OCS dose), reduced exacerbations by $32 \%$ and also improved control of asthma symptoms. In a recent phase $3 \mathrm{~b}$ placebo-controlled trial (the MUSCA study), mepolizumab was associated with significant improvement of health-related quality of life, lung function and asthma symptoms control in a large pool of patients with severe eosinophilic asthma [64]. The safety profile of mepolizumab in all published studies was similar to that of placebo $[15,55,56,64]$.

In November 2015, the US Food and Drug Administration (FDA) approved mepolizumab (administered once every 4 weeks by s.c. injection) for patients with severe eosinophilic asthma and a history of exacerbations, followed 1 month later by European Medicines Agency (EMA) approval. Mepolizumab was also incorporated in the latest GINA recommendations as an add-on treatment option in Step 5 of the treatment plan [18].

\section{Reslizumab}

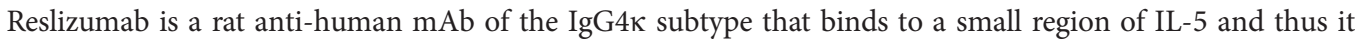
effectively blocks IL-5 from binding to the IL-5 receptor complex on the cell surface of eosinophils [61]. Reslizumab is currently in clinical trials for the treatment of hypereosinophilic syndrome, eosinophilic oesophagitis, eosinophilic granulomatosis with polyangiitis and severe eosinophilic asthma.

In the first phase 3 study published in 2011, in a cohort of 106 patients with eosinophilic asthma (sputum eosinophils $\geqslant 3 \%$ ), reslizumab significantly reduced sputum eosinophils, improved lung function and showed a trend toward better asthma control [54]. Following the paradigm of mepolizumab, reslizumab was then studied in asthma patients inadequately controlled by medium to high doses of ICSs, marked blood eosinophilia $\left(\geqslant 400\right.$ cells $\left.\mu \mathrm{L}^{-1}\right)$ and frequent exacerbations. In two duplicate, multicentre, placebo-controlled, phase 3 trials published in 2015, 953 patients were assigned to receive either i.v. reslizumab $\left(3 \mathrm{mg} \cdot \mathrm{kg}^{-1}\right)$ or placebo every 4 weeks for 1 year [57]. In both cohorts, patients receiving reslizumab had a significant reduction, of $>50 \%$, in the frequency of asthma exacerbations [57]. Finally, two studies published in 2016 confirmed previous findings, and showed that reslizumab improved lung function, asthma control, symptoms and quality of life in patients with inadequately controlled asthma and elevated blood eosinophil levels, while such clinically meaningful effects were not seen in patients unselected for baseline eosinophils $[65,66]$. Interestingly, in a recent study in 10 prednisone-dependent asthmatic subjects inadequately controlled under add-on treatment with fixed-dose mepolizumab, weight-adjusted i.v. reslizumab suppressed airway eosinophilia and improved asthma control [67]. Reslizumab was well tolerated and in all published studies the reported adverse events were similar or even fewer than that of placebo $[54,57,65,66]$.

In March 2016, the FDA approved reslizumab (administered once every 4 weeks by i.v. infusion) for patients $\geqslant 18$ years of age with asthma and elevated blood eosinophils who are inadequately controlled on ICSs. EMA approval followed a few months later.

\section{Benralizumab}

Benralizumab is a fully humanised IgG1 anti-IL-5 receptor $\alpha$ mAb derived from mice [61]. This mAb has a different mechanism of action compared with mepolizumab and reslizumab. It has an identified specificity for the human IL-5 receptor $\alpha$-subunit. By targeting the IL- 5 receptor, it prevents the binding 
of IL-5 and depletes the cells expressing the IL-5 receptor (mainly eosinophils but also basophils) by inducing apoptosis [68]. Benralizumab is currently in clinical studies in patients with asthma and COPD, as well as in eosinophilic chronic rhinosinusitis and hypereosinophilic syndrome.

In an initial phase $2 \mathrm{~b}$ dose-ranging study, efficacy and safety of benralizumab in adults with uncontrolled asthma were studied [58]. In this proof-of-concept study, benralizumab at 20 and $100 \mathrm{mg}$ doses seemed to reduce asthma exacerbations in patients with uncontrolled asthma and baseline blood eosinophils $\geqslant 300$ cells $\mu \mathrm{L}^{-1}$ [58]. Following these findings, two similar phase 3 studies have recently been published $[59,60]$. In both studies, benralizumab significantly reduced annual exacerbation rates, and improved FEV1 and asthma symptoms control, while being well tolerated $[59,60]$. In a meta-analysis of these two studies, the extent to which exacerbations rates were reduced increased with increasing blood eosinophil thresholds and with greater exacerbation history [69]. In the recently published ZONDA trial, benralizumab was effective as an oral glucocorticoid-sparing therapy in severe corticosteroid-dependent asthma associated with eosinophilia, reducing exacerbations but not affecting FEV1 [70]. Finally, the long-term safety of benralizumab will be examined in a study called BORA (ClinicalTrials.gov identifier NCT02258542), estimated to end in June 2018. Benralizumab was recently approved by the FDA and EMA for severe eosinophilic asthma treatment.

\section{Oral corticosteroids}

The proportion of severe asthmatic subjects treated with OCSs is extremely variable and is up to $60 \%$ in different cohorts, with significant geographic variation [8-10]. The dose of OCS used is also highly variable, with reports from the British Thoracic Society Severe Refractory Asthma Registry of a mean dose of prednisolone $>20 \mathrm{mg} \cdot$ day $^{-1}$ [10]. Yet, despite this widespread use, there is a relative paucity of robust study evidence proving a beneficial effect from systemic corticosteroids on exacerbation rates in severe asthmatic subjects. There are no randomised controlled trials of prednisolone versus placebo as add-on therapy in severe asthma. A large audit using data from the British Thoracic Society Difficult Asthma Registry suggested a decreased rate of severe asthma exacerbations during the follow-up period, while the proportion of patients taking OCSs increased from $42 \%$ to $57 \%$. This led the authors to suggest that maintenance OCS use was associated with a reduced rate of severe asthma exacerbations among this severe asthmatic cohort [71]. While data about efficacy are still sporadic, side-effects of chronic OCS use are quite evident. Potential adverse event reported among OCS-treated asthma patients include hypertension, diabetes, pneumonia, obesity, osteoporosis, cataracts and opportunistic infections [72].

\section{Macrolides}

Macrolides may be of benefit in asthma despite the fact that their administration may increase the risk for microbial resistance. They achieve their action through different mechanisms which are mainly related to either an anti-inflammatory effect or/and to their possible inhibitory effect on the microbiome and virobiome within the airways [73]. Only two studies have examined their clinical effect on severe asthma. The study by BRUSSELLE [73] investigated whether the administration of low-dose azithromycin in patients with severe asthma could potentially alter the rate of exacerbations during a period of 6 months follow-up. The study was considered negative since it failed to reach the primary outcome. However, a positive effect was observed in patients with noneosinophilic asthma [74]. On the contrary, the recently published AMAZES study demonstrated that adding azithromycin in a larger pool of patients with uncontrolled persistent asthma (on medium- to high-dose ICS plus LABA) reduced the exacerbation rate and improved quality of life [75]. Even so, the evidence regarding the role of macrolides in severe asthma is still very limited to support their regular use in the clinical setting.

\section{Bronchial thermoplasty}

Bronchial thermoplasty is a new bronchoscopic therapy delivering radiofrequency energy to the airways with the aim to reduce airway smooth muscle mass and smooth muscle hypertrophy [76]. Three randomised trials support the use of bronchial thermoplasty as a treatment modality for uncontrolled asthma [77-81]. Both the RISA and AIR study groups found that patients with severe asthma who underwent bronchial thermoplasty had an initial increase in hospitalisation due to respiratory adverse events, but no statistically significant difference in exacerbations in the post-treatment phase. In both studies bronchial thermoplasty was associated with an improvement in asthma control indices [77, 78]. The AIR2 study also found that bronchial thermoplasty-treated patients experienced significantly fewer asthma exacerbations, emergency department visits and days missed from work/school compared with the control (sham) arm, although the absolute difference between groups was small [79]. Follow-up data for the AIR and AIR2 studies showed that the reduction in exacerbations was maintained over a 5-year period $[80,81]$. Moreover, the results of the ongoing post-market PAS2 follow-up study at 3 years after bronchial thermoplasty confirm the AIR2 findings [82]. 
Despite the initial enthusiasm about this new treatment modality, real-life studies reported reduced rates of clinical improvement and higher rates of adverse events compared with clinical trials [83, 84]. Furthermore, this technique is an interventional procedure, not widely available, and many experts in the medical community have raised questions and concerns about its long-term efficacy and safety [85, 86]. In the latest ATS/ERS guidelines for severe asthma, it is recommended that bronchial thermoplasty must be performed in dedicated centres and only in the context of a systematic registry or a clinical study [2].

\section{Future treatments}

Despite the unmet needs in the treatment of severe asthma, very few new classes of drugs were introduced and considered safe and effective for these patients over the past decades. New therapies targeting critical mediators, receptors and enzymes implicated in the pathophysiology of severe asthma are currently under development and/or in clinical trials.

Strategies targeting the T2 axis include antibodies against IL-4, IL-13, IL-25, IL-33, thymic stromal lymphopoietin, oral CRTh2 (chemoattractant receptor-homologous molecule expressed on Th2 cells) antagonists and novel anti-IgE therapies (quilizumab, MEDI-4212, ligelizumab) [87]. Lebrikizumab is an IgG4 mAb that targets IL-13. Initial studies in moderate to severe asthma demonstrate efficacy in a subset of patients characterised by eosinophilic inflammation and high serum periostin levels [88]. However, a recent publication of two replicate phase 3 studies (LAVOLTA) failed to meet the primary outcome (reduction in the rate of asthma exacerbations in biomarker-high patients) [89]. Additionally, two phase 2 studies with tralokinumab, another IgG4 mAb that targets IL-13, failed to show clinical efficacy in patients with severe uncontrolled asthma $[90,91]$. Thus, targeting IL-13 alone might not be sufficient to provide clinically meaningful improvement in exacerbations and asthma control in these patients.

Dupilumab is a mAb directed against the $\alpha$-subunit of the IL- 4 receptor and blocks both IL- 4 and IL-13 signal transduction. In an initial phase 2 a trial, dupilumab therapy was associated with fewer asthma exacerbations when LABAs and ICS were withdrawn, with improved lung function and reduced levels of Th2-associated inflammatory markers [92]. In a following phase $2 \mathrm{~b}$ study, dupilumab treatment over a 24-week period increased lung function and reduced severe exacerbations in patients with uncontrolled persistent asthma irrespective of baseline eosinophil count, with a favourable safety profile [93]. Dupilumab seems to be a promising candidate drug for severe asthma, but larger phase 3 studies are required to establish its long-term efficacy and safety. Such studies in patients with uncontrolled and steroid-dependent asthma are currently ongoing (ClinicalTrials.gov identifiers NCT02948959 and NCT02528214).

Receptor antagonists for CRTh2 are also being investigated in asthma treatment. Fevipiprant (QAW039) is such an oral CRTh2 antagonist, and initial phase 2 studies showed some promising results in patients with airflow limitation $\left(\mathrm{FEV}_{1}<70 \%\right)$ and persistent sputum eosinophilia $[94,95]$. Two phase 3 studies with QAW039 in patients with severe asthma stratified by blood eosinophil counts are currently recruiting subjects (ClinicalTrials.gov identifiers NCT02555683 and NCT02563067).

Addressing T2-low airway inflammation with new drugs is also an interesting and challenging research area. Several novel small-molecule drugs have been developed and are currently under investigation for the treatment of neutrophilic asthma. Such molecules include C-X-C motif chemokine receptor 2 (CXCR2) antagonists, 5-lipoxygenase-activating protein inhibitors, prostaglandin $\mathrm{E}_{3} / \mathrm{E}_{4}$ and various protein kinase inhibitors [96]. Although some preliminary results, especially for CXCR2 antagonists, have been promising [14], a recent phase 2 study of AZD5069 (a CXCR2 antagonist) failed to reduce the frequency of severe exacerbations in patients with uncontrolled severe asthma [97]. Moreover, there are currently no active phase 3 clinical trials for the aforementioned drugs on ClinicalTrials.gov.

Finally, single inhaler triple therapy (LABA/LAMA/ICS) is currently under investigation in patients with uncontrolled asthma despite therapy with medium- to high-dose ICS plus LABA. Large phase 3 studies with fixed-dose combinations of fluticasone furoate plus umeclidinium bromide plus vilanterol (ClinicalTrials.gov identifier NCT02924688), beclometasone dipropionate plus formoterol fumarate plus glycopyrronium bromide (ClinicalTrials.gov identifiers NCT02676076 and NCT02676089) and indacaterol plus glycopyrronium bromide plus mometasone furoate (QVM149; ClinicalTrials.gov identifier NCT02571777) are currently ongoing. Despite the fact that none of these studies has been designed specifically for severe asthma patients, their results can still provide useful information.

\section{Treating severe asthma: an algorithmic approach}

The management of severe uncontrolled asthma in daily clinical practice is challenging for the treating physician. In the last few years, new exciting drugs have been developed and are already being used in clinical practice, while other novel approaches are currently in clinical trials. However, before resorting to 
new add-on treatment for severe uncontrolled asthma, we must not forget to re-evaluate patients' comorbidities, exposure to allergens or other noxious agents, as well as adherence and proper use of current treatment [2]. The vast majority of these patients are already on high-dose ICS/LABA fixed combination and some of them are current or former users of LAMA (tiotropium). Even if it is still not supported by current guidelines or many clinical studies, a switch to a different inhaler device (metered dose inhaler to dry powder inhaler or vice versa) or to a different ICS/LABA strategy (i.e. SMART (single maintenance and reliever therapy)) may also prove beneficial for specific uncontrolled severe asthma patients $[98,99]$. It is common knowledge that not all patients can achieve the proper technique for each type of inhaler. Therefore, rather than insisting that patients use a particular device or treatment strategy, a better way could be to match a device or treatment plan to the specific patient.

With new therapies and strategies already approved, and others upcoming in the near future, there is a clear need for a stepwise therapeutic approach in severe uncontrolled asthmatic subjects. We present a clinical algorithm (figure 1) that we currently use in our severe asthma outpatient clinics. The first step is to differentiate T2-high from T2-low asthma based on specific clinical features and biomarkers. According to the algorithm presented here, T2-high asthma is defined as the presence of atopy (SPT/RAST+) and/or IgE $>100 \mathrm{IU} \cdot \mathrm{mL}^{-1}$ and/or FENO $>30 \mathrm{ppb}$ and/or blood eosinophils $>300 \mu \mathrm{L}^{-1}$ and/or sputum eosinophils $>2 \%$. The absence of these characteristics is consistent with T2-low asthma. Periostin is also a promising biomarker for T2-high asthma, but it is not included in this algorithm as it is not yet commercially available and is only used in clinical trials.

\section{T2-high asthma endotype}

After allocating a severe asthma patient to the T2-high endotype, it is also necessary to assess which endotype, i.e. allergic or eosinophilic, is predominant. We propose the use of a set of specific clinical features and biomarkers to dissociate between these two endotypes (table 2). It is important to define the predominant endotype driving the disease in order to assess the treatment that is more likely to be successful. In clinical studies of anti-IL-5 treatment in eosinophilic asthma, "overlap" syndrome, i.e. patients sharing the obligatory characteristics of both endotypes, may be as high as $50 \%$ (table 3 ).

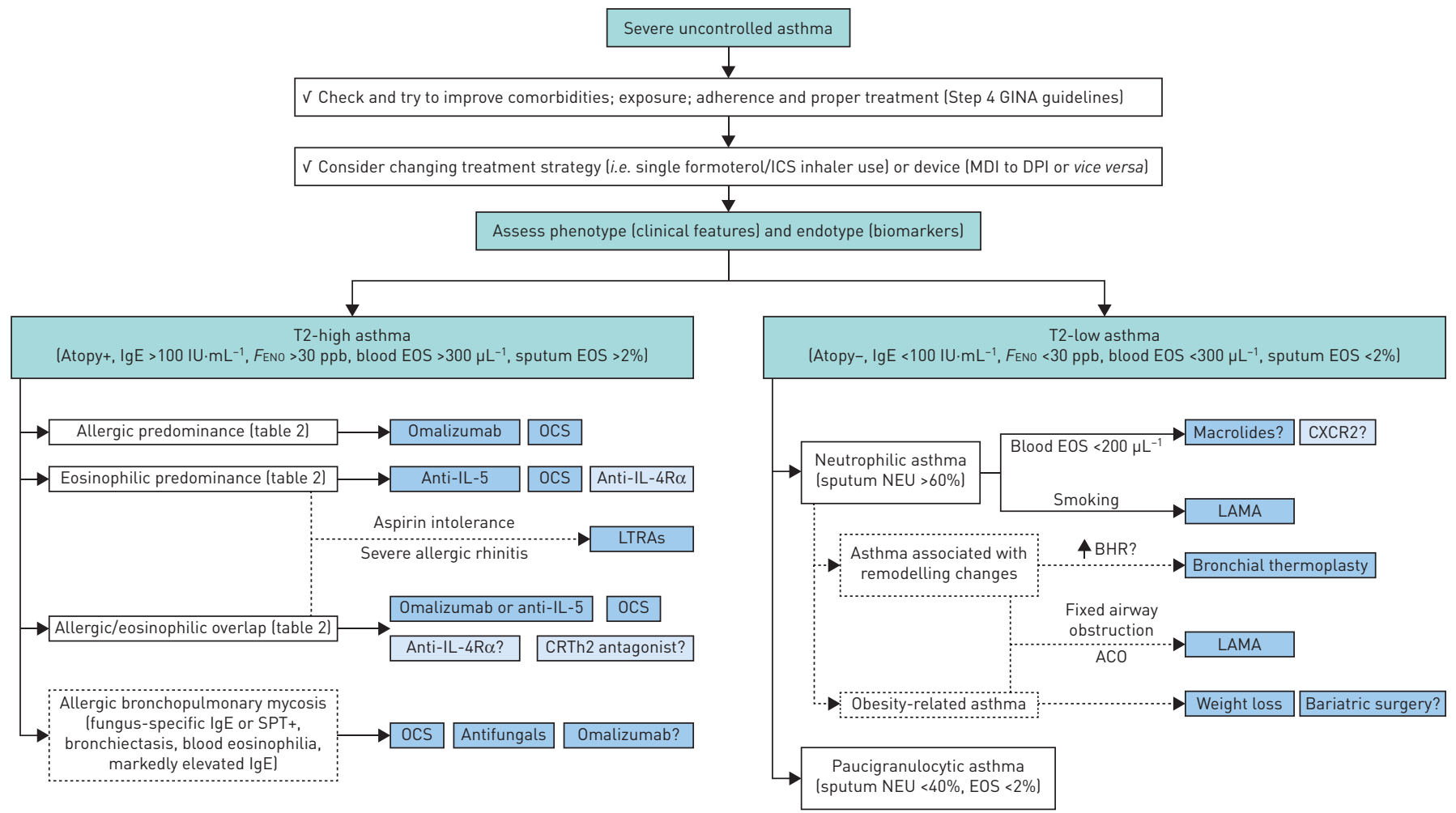

FIGURE 1 A stepwise therapeutic approach in severe uncontrolled asthmatic subjects. GINA: Global Initiative for Asthma; ICS: inhaled corticosteroid; MDI: metered dose inhaler; DPI: dry powder inhaler; FENO: exhaled nitric oxide fraction; EOS: eosinophils; OCS: oral corticosteroid; IL-5: interleukin-5; IL-4R $\alpha$ : interleukin-4 receptor $\alpha$; LTRA: leukotriene receptor antagonist; CRTh2: chemoattractant receptor-homologous molecule expressed on T-helper 2 cells; SPT: skin prick test; NEU: neutrophils; CXCR2: C-X-C motif chemokine receptor 2; LAMA: long-acting muscarinic antagonist; BHR: bronchial hyperresponsiveness; ACO: asthma-chronic obstructive pulmonary disease overlap. Dark blue boxes correspond to currently available treatment options; light blue boxes correspond to future treatments. 


\begin{tabular}{|c|c|c|}
\hline & $\begin{array}{c}\text { A: } \\
\text { allergic-predominant asthma }\end{array}$ & $\begin{array}{c}\text { B: } \\
\text { eosinophilic-predominant asthma }\end{array}$ \\
\hline 1 & Early onset & Late onset \\
\hline 2 & $\begin{array}{c}\text { SPT/RAST+ with clinically significant } \\
\text { allergies }{ }^{\#}\end{array}$ & $\begin{array}{c}\text { SPT/RAST- or + with no clinically significant } \\
\text { allergies }\end{array}$ \\
\hline 3 & $\lg E>100 \mathrm{IU} \cdot \mathrm{mL}^{-1}$ & $\lg \mathrm{E}<100 \mathrm{IU} \cdot \mathrm{mL}^{-1}$ \\
\hline 4 & Allergic rhinitis & Nasal polyps \\
\hline 5 & High FENO $(30-50 \mathrm{ppb})$ & Very high FENO $(>50 \mathrm{ppb})$ \\
\hline 6 & Blood eosinophils $<300$ cells $\cdot \mu \mathrm{L}^{-1}$ & Blood eosinophils $>300$ cells $\cdot \mu \mathrm{L}^{-1 \#}$ \\
\hline \multicolumn{3}{|c|}{$\begin{array}{l}\text { SPT: skin prick test; RAST: radioallergosorbent test; FENO: exhaled nitric oxide fraction. Check the number } \\
\text { of relevant patient characteristics per column. If a patient has more features from column A or B it is } \\
\text { more likely that he/she has allergic- or eosinophilic-predominant asthma, respectively. If the patient } \\
\text { shares features from both columns, it is more likely that he/she suffers from eosinophilic/allergic overlap } \\
\text { asthma. " obligatory characteristics for allergic and/or eosinophilic asthma. }\end{array}$} \\
\hline
\end{tabular}

Using the approach proposed in table 2 in everyday clinical practice, the vast majority of patients can be allocated to either allergic- or eosinophilic-predominant T2-high asthma. In allergic-predominant severe asthma, omalizumab is the treatment of choice, while in eosinophilic-predominant asthma, anti-IL-5 treatment is the reasonable therapeutic approach. From our experience, also supported by recent studies, only a minority of patients, probably $<20 \%$, share equal features from both groups and no predominant endotype is evident [100]. In these patients with an overlapping phenotype, the treating physician may choose to start with either anti-IgE or anti-IL-5 treatment [101]. Given that omalizumab has established

TABLE 3 Summary of randomised controlled trials with anti-interleukin-5 treatment: eosinophil threshold used in each trial and percentage of atopics in enrolled patients

\begin{tabular}{|c|c|c|c|c|c|}
\hline First author [ref.] & Year & Treatment & Eosinophil threshold & Patients $\mathbf{n}$ & Atopic \\
\hline Haldar [24] & 2009 & Mepolizumab & $>3 \%$ sputum eosinophils & 61 & $68 \% \mathrm{SPT}+$ \\
\hline Castro [54] & 2011 & Reslizumab & $>3 \%$ sputum eosinophils & 106 & $\begin{array}{l}80 \% \text { allergic } \\
\text { rhinitis }\end{array}$ \\
\hline Pavord [15] & 2012 & Mepolizumab & $>300 \mu \mathrm{L}^{-1}$ blood eosinophils & 616 & $50 \%$ SPT+ \\
\hline Ortega [55] & 2014 & Mepolizumab & $\begin{array}{c}>150 \mu \mathrm{L}^{-1} \text { blood eosinophils } \\
\text { at screening or }>300 \mu \mathrm{L}^{-1} \\
\text { during previous year }\end{array}$ & 576 & $\begin{array}{c}49 \% \text { allergic } \\
\text { rhinitis }\end{array}$ \\
\hline Bel [56] & 2014 & Mepolizumab & $>300 \mu \mathrm{L}^{-1}$ blood eosinophils & 135 & $\begin{array}{l}46 \% \text { allergic } \\
\text { rhinitis }\end{array}$ \\
\hline Castro [58] & 2014 & Benralizumab & $\begin{array}{l}\text { No threshold for } \\
\text { eosinophils; FENO >50 ppb } \\
\text { used as surrogate marker }\end{array}$ & 244 & Not reported \\
\hline Castro [57] & 2015 & Reslizumab & $>400 \mu \mathrm{L}^{-1}$ blood eosinophils & 953 & Not reported \\
\hline Corren [66] & 2016 & Reslizumab & $\begin{array}{l}\text { No initial threshold for } \\
\text { eosinophils; } \geqslant 400 \mu \mathrm{L}^{-1} \text { blood } \\
\text { eosinophils as clinically } \\
\text { significant }\end{array}$ & 492 & Not reported \\
\hline Bjermer [65] & 2016 & Reslizumab & $\geqslant 400 \mu \mathrm{L}^{-1}$ blood eosinophils & 315 & Not reported \\
\hline FitzGerald [60] & 2016 & Benralizumab & $\begin{array}{l}\text { No initial threshold for } \\
\text { eosinophils; } \geqslant 300 \mu \mathrm{L}^{-1} \text { blood } \\
\text { eosinophils as clinically } \\
\text { significant }\end{array}$ & 1306 & $62 \%$ RAST+ \\
\hline Bleecker [59] & 2016 & Benralizumab & $\begin{array}{l}\text { No initial threshold for } \\
\text { eosinophils; } \geqslant 300 \mu L^{-1} \text { blood } \\
\text { eosinophils as clinically } \\
\text { significant }\end{array}$ & 1204 & $63 \%$ RAST+ \\
\hline
\end{tabular}


efficacy and safety data for $>10$ years, and doctors have more experience using it, omalizumab is more likely to be the starting treatment in these patients. However, mepolizumab or benralizumab could be considered as a first-choice treatment in patients with corticosteroid-dependent asthma, based on data from randomised clinical trials showing efficacy and an oral steroid-sparing effect in this specific group of patients $[56,70]$. So, in this overlapping patient population there is no objective and evidence-based answer to the question: "Anti-IgE or anti-IL-5 as a first choice?", as no direct comparison between these two treatments was ever made or planned and probably will never be done.

Irrespectively of the initial treatment, patients should be frequently re-evaluated and in case of lack of improvement, a switch of treatment could be considered. In favour of this, recent data suggest that mepolizumab has similar efficacy even in patients previously treated with omalizumab, while data on the opposite approach are not available [102]. A definite answer to this approach, at least for the switch from omalizumab to mepolizumab, could be expected from an ongoing multicentre randomised controlled trial that aims to evaluate the effect of mepolizumab in patients with severe eosinophilic asthma not optimally controlled with omalizumab (ClinicalTrials.gov identifier NCT02654145).

Regarding anti-IL-5 treatment, the level of blood eosinophils that accurately selects possible responders, the optional duration of treatment and the safety of the long-term use of these drugs remain to be defined. Different biomarkers and cut-off values have been reported in studies (table 3). Moreover, to date, little is known about the effects of withdrawal of anti-IL-5 treatment. HALDAR et al. [103] reported outcomes after cessation of mepolizumab therapy in severe eosinophilic asthma over a 12-month follow-up period. Cessation of mepolizumab was associated with a rise in the blood eosinophil count, returning to baseline levels over 6 months. Furthermore, the frequency of severe exacerbations as well as asthma symptoms increased significantly after stopping mepolizumab [103]. These findings support the long-term or even the life-long use of anti-IL-5 treatment in order to maintain suppression of eosinophilic airway inflammation. As for the long-term data, these are still awaited.

An alternative treatment option in this T2-high group of patients, irrespective of eosinophilic or allergic endotype, is the use of OCSs. This treatment approach has been used for $>30$ years now, but with relative low efficacy and disproportional side-effects [72]. However, when treatment with mAbs is not effective, available or afforded/reimbursed, prednisolone remains the treatment of choice for this T2-high group of patients. The dose needed to retain control of the disease must be individualised and, as stated in the latest GINA recommendations [18] and in our clinical experience, 5-10 mg daily is effective for the vast majority of these patients.

In a small minority of T2-high severe uncontrolled asthma with overexpressed Th2 inflammation (IgE $>1000 \mathrm{IU} \cdot \mathrm{mL}^{-1}$ and blood eosinophils $>500 \mu \mathrm{L}^{-1}$ ), the allergic bronchopulmonary mycosis (ABPM) endotype must be considered. Cough and phlegm are generally the main manifestation of the disease, and these symptoms are usually associated with bronchiectasis on lung imaging. Proof of sensitisation to Aspergillus or other fungi is necessary for this endotype diagnosis, with positive immediate skin test or increased specific IgE antibody to the responsible fungi [104]. Primary therapy consists of OCSs and many of these patients will require life-long treatment with these drugs. Antifungals can be used as a steroid-sparing agent, and some small placebo-controlled studies in allergic bronchopulmonary aspergillosis (ABPA) have reported efficacy of itraconazole in symptoms control and reduction of exacerbations $[105,106]$. Numerous case series suggest that next-generation antifungals may be more efficacious, although a recent randomised placebo-controlled trial with voriconazole failed to show a beneficial effect in the treatment of Aspergillus fumigatus-associated asthma [107]. The only Th2-targeted approach that has been reported in this endotype is the use of anti-IgE treatment. A recent review of 102 cases from 30 published studies [108], as well as the first randomised placebo-controlled trial of omalizumab therapy in ABPA [109], provide evidence for the efficacy and safety of this therapeutic intervention in ABPM. It must, however, be emphasised that the use of omalizumab in this T2-high endotype is currently off-label.

Finally, in some T2-high severe uncontrolled patients where the clinical presentation suggests that leukotrienes may be strongly implicated in the underlying pathophysiology (i.e. aspirin sensitivity, concomitant severe allergic rhinitis), a therapeutic trial with LTRAs could be considered [110, 111]. Regarding future treatments and drugs in the pipeline, the most promising data are from dupilumab and fevipiprant (QAW039) studies, but results from phase 3 studies are still lacking.

\section{T2-low asthma endotype}

T2-low disease was initially considered to be a rather rare entity in the context of severe asthma; however, recent data indicate that it may affect up to a third of severe asthmatic subjects [9]. The lack of an effective controller medication for patients with T2-low asthma is a substantial clinical problem that currently has 
no clear solution. Assessing airway inflammation by sputum differential cell counts is not widely available in clinical practice, so we used other surrogate markers or disease characteristics in our therapeutic algorithm (figure 1).

Smoking is a well-known factor that not only aggravates asthma symptoms and worsens asthma control, but is also associated with neutrophilic asthma that is refractory to corticosteroids and other medication [112]. Asthma-COPD overlap is also related to smoking and as the percentage of current or ex-smokers in severe asthma cohorts remains high (30-50\%) [8-10], this diagnosis must be always considered. Asthma-COPD overlap is also associated with fixed airway obstruction and lower FEV1, features of prominent remodelling changes, which are common characteristics in this group of T2-low asthma endotype. Tiotropium (soft-mist inhaler) is the only LAMA formulation that has been recently included as a new add-on asthma treatment for Steps 4 and 5 in the GINA recommendations [18], but also all the other LAMAs can be used in the case of COPD comorbidity [18]. In this subgroup of severe T2-low asthmatic subjects, LAMAs (if not previously used) seem to be the next logical add-on treatment (figure 1).

The "off-label" use of macrolides is an alternative therapeutic option for some T2-low asthmatic subjects, as suggested by subgroup analysis in the study by BRUSSELlE et al. [74]. Current smokers were excluded from the positive AMAZES study [75] and a randomised controlled trial of azithromycin in smokers with asthma failed to demonstrate efficacy in any clinical or laboratory outcome [113]. Therefore, in nonsmoking T2-low severe asthmatic subjects, a trial with low-dose azithromycin $\left(250 \mathrm{mg} \cdot\right.$ day $^{-1}$ for 5 days and then one capsule three times a week for 3 months) can be considered as a treatment option (figure 1).

Other treatment approaches currently available for T2-low asthma address noninflammatory pathways possibly involved in its pathogenesis. A therapeutic approach for patients with prominent remodelling changes, mainly smooth muscle hypertrophy, is bronchial thermoplasty. As this technique is invasive and not widely available, it is proposed that bronchial thermoplasty must be performed in experienced centres and only in the context of a systematic registry. Obesity-associated asthma represents another distinct clinical phenotype often associated with the T2-low endotype in worldwide registries of severe asthma [8, 9, 114]. Several studies have shown that weight loss in obese adults with severe asthma can improve asthma control, lung function, airway hyperresponsiveness and quality of life $[115,116]$. These findings support the need to actively pursue healthy weight-loss measures in this phenotype as the first management option (figure 1). Moreover, a recent well-designed study reports positive effects of bariatric surgery on asthma control, lung function, and bronchial and systemic inflammation in morbidly obese mild to moderate asthmatic subjects [117]. Case reports also show favourable results in severe uncontrolled asthmatic subjects $[118,119]$. These data allow us to consider this invasive surgical approach for weight loss in this asthma phenotype, if other weight-loss measures have failed (figure 1).

Regarding future treatments, several novel small-molecule drugs have been developed and are currently under investigation for the treatment of neutrophilic asthma, but none has yet reached phase 3 trials [96]. New treatment options for T2-low asthma are urgently needed.

\section{Conclusions}

Management of severe uncontrolled asthma is difficult and challenging. Understanding the patient's phenotype characteristics and identifying biomarkers can help us classify the underlying disease endotype and address appropriate therapy in an algorithmic stepwise approach. Treatment options in severe asthma have been enriched in recent years, but unmet needs still exist, especially in T2-low asthma.

\section{References}

1 Gaga M, Zervas E, Samitas K, et al. Severe asthma in adults: an orphan disease? Clin Chest Med 2012; 33: $571-583$.

2 Chung KF, Wenzel SE, Brozek JL, et al. International ERS/ATS guidelines on definition, evaluation and treatment of severe asthma. Eur Respir J 2014; 43: 343-373.

3 The ENFUMOSA Study Group. The ENFUMOSA cross-sectional European multicentre study of the clinical phenotype of chronic severe asthma. Eur Respir J 2003; 22: 470-477.

4 Kupczyk M, ten Brinke A, Sterk PJ, et al. Frequent exacerbators - a distinct phenotype of severe asthma. Clin Exp Allergy 2014; 44: 212-221.

5 Kupczyk M, Dahlen B, Sterk PJ, et al. Stability of phenotypes defined by physiological variables and biomarkers in adults with asthma. Allergy 2014; 69: 1198-1204.

6 Osborne M, Deffebach M. The Epidemiology and Natural History of Asthma: Outcomes and Treatment Regimens (TENOR) study. Ann Allergy Asthma Immunol 2004; 92: 3-4.

7 Jarjour NN, Erzurum SC, Bleecker ER, et al. Severe asthma: lessons learned from the National Heart, Lung, and Blood Institute Severe Asthma Research Program. Am J Respir Crit Care Med 2012; 185: 356-362.

8 Moore WC, Meyers DA, Wenzel SE, et al. Identification of asthma phenotypes using cluster analysis in the Severe Asthma Research Program. Am J Respir Crit Care Med 2010; 181: 315-323. 

Asthma Registry (BSAR). Respir Med 2014; 108: 1723-1732.

10 Newby C, Heaney LG, Menzies-Gow A, et al. Statistical cluster analysis of the British Thoracic Society Severe Refractory Asthma Registry: clinical outcomes and phenotype stability. PLoS One 2014; 9: e102987.

11 Bel EH, Sousa A, Fleming L, et al. Diagnosis and definition of severe refractory asthma: an international consensus statement from the Innovative Medicine Initiative (IMI). Thorax 2011; 66: 910-917.

12 Hanania NA, Alpan O, Hamilos DL, et al. Omalizumab in severe allergic asthma inadequately controlled with standard therapy: a randomized trial. Ann Intern Med 2011; 154: 573-582.

13 Nair P, Pizzichini MM, Kjarsgaard M, et al. Mepolizumab for prednisone-dependent asthma with sputum eosinophilia. N Engl J Med 2009; 360: 985-993.

14 Nair P, Gaga M, Zervas E, et al. Safety and efficacy of a CXCR2 antagonist in patients with severe asthma and sputum neutrophils: a randomized, placebo-controlled clinical trial. Clin Exp Allergy 2012; 42: 1097-1103.

15 Pavord ID, Korn S, Howarth P, et al. Mepolizumab for severe eosinophilic asthma (DREAM): a multicentre, double-blind, placebo-controlled trial. Lancet 2012; 380: 651-659.

16 National Asthma Education and Prevention Program Expert Panel Report 2. Guidelines for the Diagnosis and Management of Asthma. Bethesda, National Heart, Lung, and Blood Institute, 1997.

17 Global Initiative for Asthma. Asthma Management and Prevention. NIH Publication 95-3659A. Bethesda, National Institutes of Health, 1995.

18 Global Initiative for Asthma. Global Strategy for Asthma Management and Prevention. 2017. http://ginasthma. org/2017-gina-report-global-strategy-for-asthma-management-and-prevention Date last accessed: November 15, 2017.

19 National Asthma Education and Prevention Program. Expert Panel Report 3 (EPR-3): Guidelines for the Diagnosis and Management of Asthma - Summary Report 2007. J Allergy Clin Immunol 2007; 120: S94-S138.

20 American Thoracic Society. Proceedings of the ATS workshop on refractory asthma: current understanding, recommendations, and unanswered questions. Am J Respir Crit Care Med 2000; 162: 2341-2351.

21 Chanez P, Wenzel SE, Anderson GP, et al. Severe asthma in adults: what are the important questions? J Allergy Clin Immunol 2007; 119: 1337-1348.

22 Chung KF, Godard P, Adelroth E, et al. Difficult/therapy-resistant asthma: the need for an integrated approach to define clinical phenotypes, evaluate risk factors, understand pathophysiology and find novel therapies. ERS Task Force on Difficult/Therapy-Resistant Asthma. Eur Respir J 1999; 13: 1198-1208.

23 Reddel HK, Taylor DR, Bateman ED, et al. An official American Thoracic Society/European Respiratory Society statement: asthma control and exacerbations: standardizing endpoints for clinical asthma trials and clinical practice. Am J Respir Crit Care Med 2009; 180: 59-99.

24 Haldar P, Brightling CE, Hargadon B, et al. Mepolizumab and exacerbations of refractory eosinophilic asthma. N Engl J Med 2009; 360: 973-984.

25 Katz LE, Gleich GJ, Hartley BF, et al. Blood eosinophil count is a useful biomarker to identify patients with severe eosinophilic asthma. Ann Am Thorac Soc 2014; 11: 531-536.

26 Ortega HG, Yancey SW, Mayer B, et al. Severe eosinophilic asthma treated with mepolizumab stratified by baseline eosinophil thresholds: a secondary analysis of the DREAM and MENSA studies. Lancet Respir Med 2016; 4: 549-556.

27 Haldar P, Pavord ID. Noneosinophilic asthma: a distinct clinical and pathologic phenotype. J Allergy Clin Immunol 2007; 119: 1043-1052.

28 Parulekar AD, Diamant Z, Hanania NA. Role of T2 inflammation biomarkers in severe asthma. Curr Opin Pulm Med 2016; 22: 59-68.

29 Busse WW, Holgate ST, Wenzel SW, et al. Biomarker profiles in asthma with high vs low airway reversibility and poor disease control. Chest 2015; 148: 1489-1496.

30 Disse B, Speck GA, Rominger KL, et al. Tiotropium (Spiriva): mechanistical considerations and clinical profile in obstructive lung disease. Life Sci 1999; 64: 457-464.

31 Bos IS, Gosens R, Zuidhof AB, et al. Inhibition of allergen-induced airway remodelling by tiotropium and budesonide: a comparison. Eur Respir J 2007; 30: 653-661.

32 Gosens R, Bos IS, Zaagsma J, et al. Protective effects of tiotropium bromide in the progression of airway smooth muscle remodeling. Am J Respir Crit Care Med 2005; 171: 1096-1102.

33 Price D, Fromer L, Kaplan A, et al. Is there a rationale and role for long-acting anticholinergic bronchodilators in asthma? NPJ Prim Care Respir Med 2014; 24: 14023.

34 Kerstjens HA, Disse B, Schroder-Babo W, et al. Tiotropium improves lung function in patients with severe uncontrolled asthma: a randomized controlled trial. J Allergy Clin Immunol 2011; 128: 308-314.

35 Kerstjens HA, Engel M, Dahl R, et al. Tiotropium in asthma poorly controlled with standard combination therapy. N Engl J Med 2012; 367: 1198-1207.

36 Price D, Kaplan A, Jones R, et al. Long-acting muscarinic antagonist use in adults with asthma: real-life prescribing and outcomes of add-on therapy with tiotropium bromide. J Asthma Allergy 2015; 8: 1-13.

37 Ishizaka K, Ishizaka T. Identification of gamma-E-antibodies as a carrier of reaginic activity. J Immunol 1967; 99: 1187-1198.

38 Fahy JV, Fleming HE, Wong HH, et al. The effect of an anti-IgE monoclonal antibody on the early- and late-phase responses to allergen inhalation in asthmatic subjects. Am J Respir Crit Care Med 1997; 155: $1828-1834$

39 Bousquet J, Cabrera P, Berkman N, et al. The effect of treatment with omalizumab, an anti-IgE antibody, on asthma exacerbations and emergency medical visits in patients with severe persistent asthma. Allergy 2005; 60: 302-308.

40 Holgate ST, Chuchalin AG, Hebert J, et al. Efficacy and safety of a recombinant anti-immunoglobulin E antibody (omalizumab) in severe allergic asthma. Clin Exp Allergy 2004; 34: 632-638.

41 Humbert M, Beasley R, Ayres J, et al. Benefits of omalizumab as add-on therapy in patients with severe persistent asthma who are inadequately controlled despite best available therapy (GINA 2002 step 4 treatment): INNOVATE. Allergy 2005; 60: 309-316. 
Tzortzaki EG, Georgiou A, Kampas D, et al. Long-term omalizumab treatment in severe allergic asthma: the South-Eastern Mediterranean "real-life" experience. Pulm Pharmacol Ther 2012; 25: 77-82.

43 Vennera MC, Perez De LL, Bardagi S, et al. Omalizumab therapy in severe asthma: experience from the Spanish registry - some new approaches. J Asthma 2012; 49: 416-422.

44 Barnes N, Menzies-Gow A, Mansur AH, et al. Effectiveness of omalizumab in severe allergic asthma: a retrospective UK real-world study. J Asthma 2013; 50: 529-536.

45 Abraham I, Alhossan A, Lee CS, et al. 'Real-life' effectiveness studies of omalizumab in adult patients with severe allergic asthma: systematic review. Allergy 2016; 71: 593-610.

46 Long A, Rahmaoui A, Rothman KJ, et al. Incidence of malignancy in patients with moderate-to-severe asthma treated with or without omalizumab. J Allergy Clin Immunol 2014; 134: 560-567.

47 Bousquet J, Wenzel S, Holgate S, et al. Predicting response to omalizumab, an anti-IgE antibody, in patients with allergic asthma. Chest 2004; 125: 1378-1386.

48 Hanania NA, Wenzel S, Rosen K, et al. Exploring the effects of omalizumab in allergic asthma: an analysis of biomarkers in the EXTRA study. Am J Respir Crit Care Med 2013; 187: 804-811.

49 Slavin RG, Ferioli C, Tannenbaum SJ, et al. Asthma symptom re-emergence after omalizumab withdrawal correlates well with increasing IgE and decreasing pharmacokinetic concentrations. J Allergy Clin Immunol 2009; 123: 107-113.

50 Ledford D, Busse W, Trzaskoma B, et al. A randomized multicenter study evaluating Xolair persistence of response after long-term therapy. J Allergy Clin Immunol 2017; 140: 162-169.

51 Egan RW, Umland SP, Cuss FM, et al. Biology of interleukin-5 and its relevance to allergic disease. Allergy 1996; 51: $71-81$

52 Bousquet J, Chanez P, Lacoste JY, et al. Eosinophilic inflammation in asthma. N Engl J Med 1990; 323: 1033-1039.

53 Menzies-Gow A, Flood-Page P, Sehmi R, et al. Anti-IL-5 (mepolizumab) therapy induces bone marrow eosinophil maturational arrest and decreases eosinophil progenitors in the bronchial mucosa of atopic asthmatics. J Allergy Clin Immunol 2003; 111: 714-719.

54 Castro M, Mathur S, Hargreave F, et al. Reslizumab for poorly controlled, eosinophilic asthma: a randomized, placebo-controlled study. Am J Respir Crit Care Med 2011; 184: 1125-1132.

55 Ortega HG, Liu MC, Pavord ID, et al. Mepolizumab treatment in patients with severe eosinophilic asthma. $N$ Engl J Med 2014; 371: 1198-1207.

56 Bel EH, Wenzel SE, Thompson PJ, et al. Oral glucocorticoid-sparing effect of mepolizumab in eosinophilic asthma. N Engl J Med 2014; 371: 1189-1197.

57 Castro M, Zangrilli J, Wechsler ME, et al. Reslizumab for inadequately controlled asthma with elevated blood eosinophil counts: results from two multicentre, parallel, double-blind, randomised, placebo-controlled, phase 3 trials. Lancet Respir Med 2015; 3: 355-366.

58 Castro M, Wenzel SE, Bleecker ER, et al. Benralizumab, an anti-interleukin 5 receptor alpha monoclonal antibody, versus placebo for uncontrolled eosinophilic asthma: a phase $2 \mathrm{~b}$ randomised dose-ranging study. Lancet Respir Med 2014; 2: 879-890.

59 Bleecker ER, FitzGerald JM, Chanez P, et al. Efficacy and safety of benralizumab for patients with severe asthma uncontrolled with high-dosage inhaled corticosteroids and long-acting $\beta_{2}$-agonists (SIROCCO): a randomised, multicentre, placebo-controlled phase 3 trial. Lancet 2016; 388: 2115-2127.

60 FitzGerald JM, Bleecker ER, Nair P, et al. Benralizumab, an anti-interleukin-5 receptor alpha monoclonal antibody, as add-on treatment for patients with severe, uncontrolled, eosinophilic asthma (CALIMA): a randomised, double-blind, placebo-controlled phase 3 trial. Lancet 2016; 388: 2128-2141.

61 Samitas K, Radinger M, Bossios A. Current update on eosinophilic lung diseases and anti-IL-5 treatment. Recent Pat Antiinfect Drug Discov 2011; 6: 189-205.

62 Flood-Page PT, Menzies-Gow AN, Kay AB, et al. Eosinophil's role remains uncertain as anti-interleukin-5 only partially depletes numbers in asthmatic airway. Am J Respir Crit Care Med 2003; 167: 199-204.

63 Flood-Page P, Swenson C, Faiferman I, et al. A study to evaluate safety and efficacy of mepolizumab in patients with moderate persistent asthma. Am J Respir Crit Care Med 2007; 176: 1062-1071.

64 Chupp GL, Bradford ES, Albers FC, et al. Efficacy of mepolizumab add-on therapy on health-related quality of life and markers of asthma control in severe eosinophilic asthma (MUSCA): a randomised, double-blind, placebo-controlled, parallel-group, multicentre, phase 3b trial. Lancet Respir Med 2017; 5: 390-400.

65 Bjermer L, Lemiere C, Maspero J, et al. Reslizumab for inadequately controlled asthma with elevated blood eosinophil levels: a randomized phase 3 study. Chest 2016; 150: 789-798.

66 Corren J, Weinstein S, Janka L, et al. Phase 3 study of reslizumab in patients with poorly controlled asthma: effects across a broad range of eosinophil counts. Chest 2016; 150: 799-810.

67 Mukherjee M, Aleman PF, Kjarsgaard M, et al. Weight-adjusted intravenous reslizumab in severe asthma with inadequate response to fixed-dose subcutaneous mepolizumab. Am J Respir Crit Care Med 2018; 197: 38-46.

68 Kolbeck R, Kozhich A, Koike M, et al. MEDI-563, a humanized anti-IL-5 receptor $\alpha$ mAb with enhanced antibody-dependent cell-mediated cytotoxicity function. J Allergy Clin Immunol 2010; 125: 1344-1353

69 FitzGerald JM, Bleecker ER, Menzies-Gow A, et al. Predictors of enhanced response with benralizumab for patients with severe asthma: pooled analysis of the SIROCCO and CALIMA studies. Lancet Respir Med 2018; 6: 51-64.

70 Nair P, Wenzel S, Rabe KF, et al. Oral glucocorticoid-sparing effect of benralizumab in severe asthma. $N$ Engl $J$ Med 2017; 376: 2448-2458.

71 Sweeney J, Brightling CE, Menzies-Gow A, et al. Clinical management and outcome of refractory asthma in the UK from the British Thoracic Society Difficult Asthma Registry. Thorax 2012; 67: 754-756.

72 Zazzali JL, Broder MS, Omachi TA, et al. Risk of corticosteroid-related adverse events in asthma patients with high oral corticosteroid use. Allergy Asthma Proc 2015; 36: 268-274.

73 Brusselle GG. Are the antimicrobial properties of macrolides required for their therapeutic efficacy in chronic neutrophilic airway diseases? Thorax 2015; 70: 401-403.

74 Brusselle GG, Vanderstichele C, Jordens P, et al. Azithromycin for prevention of exacerbations in severe asthma (AZISAST): a multicentre randomised double-blind placebo-controlled trial. Thorax 2013; 68: 322-329. 

adults with persistent uncontrolled asthma (AMAZES): a randomised, double-blind, placebo-controlled trial. Lancet 2017; 390: 659-668.

76 Trivedi A, Pavord ID, Castro M. Bronchial thermoplasty and biological therapy as targeted treatments for severe uncontrolled asthma. Lancet Respir Med 2016; 4: 585-592.

77 Cox G, Thomson NC, Rubin AS, et al. Asthma control during the year after bronchial thermoplasty. $N$ Engl J Med 2007; 356: 1327-1337.

78 Pavord ID, Cox G, Thomson NC, et al. Safety and efficacy of bronchial thermoplasty in symptomatic, severe asthma. Am J Respir Crit Care Med 2007; 176: 1185-1191.

79 Castro M, Rubin AS, Laviolette M, et al. Effectiveness and safety of bronchial thermoplasty in the treatment of severe asthma: a multicenter, randomized, double-blind, sham-controlled clinical trial. Am J Respir Crit Care Med 2010; 181: 116-124

80 Thomson NC, Rubin AS, Niven RM, et al. Long-term (5 year) safety of bronchial thermoplasty: Asthma Intervention Research (AIR) trial. BMC Pulm Med 2011; 11: 8 .

81 Wechsler ME, Laviolette M, Rubin AS, et al. Bronchial thermoplasty: long-term safety and effectiveness in patients with severe persistent asthma. J Allergy Clin Immunol 2013; 132: 1295-1302.

82 Chupp G, Laviolette M, Cohn L, et al. Long-term outcomes of bronchial thermoplasty in subjects with severe asthma: a comparison of 3-year follow-up results from two prospective multicentre studies. Eur Respir J 2017; 50: 1700017.

83 Bicknell S, Chaudhuri R, Lee N, et al. Effectiveness of bronchial thermoplasty in severe asthma in 'real life' patients compared with those recruited to clinical trials in the same centre. Ther Adv Respir Dis 2015; 9 ; 267-271.

84 Burn J, Sims AJ, Keltie K, et al. Procedural and short-term safety of bronchial thermoplasty in clinical practice: evidence from a national registry and Hospital Episode Statistics. J Asthma 2017; 54: 872-879.

85 Iyer VN, Lim KG. Bronchial thermoplasty: reappraising the evidence (or lack thereof). Chest 2014; 146: 17-21.

86 Ibrahim W. Long-term efficacy and safety of bronchial thermoplasty in patients with moderate to severe persistent asthma: reproducing the same mistake. J Asthma 2016; 53: 457.

87 Sulaiman I, Lim JC, Soo HL, et al. Molecularly targeted therapies for asthma: current development, challenges and potential clinical translation. Pulm Pharmacol Ther 2016; 40: 52-68.

88 Hanania NA, Noonan M, Corren J, et al. Lebrikizumab in moderate-to-severe asthma: pooled data from two randomised placebo-controlled studies. Thorax 2015; 70: 748-756.

89 Hanania NA, Korenblat P, Chapman KR, et al. Efficacy and safety of lebrikizumab in patients with uncontrolled asthma (LAVOLTA I and LAVOLTA II): replicate, phase 3, randomised, double-blind, placebo-controlled trials. Lancet Respir Med 2016; 4: 781-796.

90 Piper E, Brightling C, Niven R, et al. A phase II placebo-controlled study of tralokinumab in moderate-to-severe asthma. Eur Respir J 2013; 41: 330-338.

91 Brightling CE, Chanez P, Leigh R, et al. Efficacy and safety of tralokinumab in patients with severe uncontrolled asthma: a randomised, double-blind, placebo-controlled, phase $2 \mathrm{~b}$ trial. Lancet Respir Med 2015; 3: 692-701.

92 Wenzel S, Ford L, Pearlman D, et al. Dupilumab in persistent asthma with elevated eosinophil levels. $N$ Engl J Med 2013; 368: 2455-2466.

93 Wenzel S, Castro M, Corren J, et al. Dupilumab efficacy and safety in adults with uncontrolled persistent asthma despite use of medium-to-high-dose inhaled corticosteroids plus a long-acting $\beta_{2}$ agonist: a randomised double-blind placebo-controlled pivotal phase $2 \mathrm{~b}$ dose-ranging trial. Lancet 2016; 388: 31-44.

94 Erpenbeck VJ, Popov TA, Miller D, et al. The oral CRTh2 antagonist QAW039 (fevipiprant): a phase II study in uncontrolled allergic asthma. Pulm Pharmacol Ther 2016; 39: 54-63.

95 Gonem S, Berair R, Singapuri A, et al. Fevipiprant, a prostaglandin $\mathrm{D}_{2}$ receptor 2 antagonist, in patients with persistent eosinophilic asthma: a single-centre, randomised, double-blind, parallel-group, placebo-controlled trial. Lancet Respir Med 2016; 4: 699-707.

96 Samitas K, Zervas E, Gaga M. T2-low asthma: current approach to diagnosis and therapy. Curr Opin Pulm Med 2017; 23: 48-55.

97 O’Byrne PM, Metev H, Puu M, et al. Efficacy and safety of a CXCR2 antagonist, AZD5069, in patients with uncontrolled persistent asthma: a randomised, double-blind, placebo-controlled trial. Lancet Respir Med 2016; 4: 797-806.

98 Aalbers R, Vogelmeier C, Kuna P. Achieving asthma control with ICS/LABA: a review of strategies for asthma management and prevention. Respir Med 2016; 111: 1-7.

99 Haughney J, Price D, Barnes NC, et al. Choosing inhaler devices for people with asthma: current knowledge and outstanding research needs. Respir Med 2010; 104: 1237-1245.

100 Albers FC, Mullerova H, Gunsoy NB, et al. Biologic treatment eligibility for real-world patients with severe asthma: the IDEAL study. J Asthma 2018; 55: 152-160.

101 Papathanassiou E, Loukides S, Bakakos P. Severe asthma: anti-IgE or anti-IL-5? Eur Clin Respir J 2016; 3: 31813.

102 Prazma CM, Wenzel S, Barnes N, et al. Characterisation of an OCS-dependent severe asthma population treated with mepolizumab. Thorax 2014; 69: 1141-1142.

103 Haldar P, Brightling CE, Singapuri A, et al. Outcomes after cessation of mepolizumab therapy in severe eosinophilic asthma: a 12-month follow-up analysis. J Allergy Clin Immunol 2014; 133: 921-923.

104 Agarwal R, Chakrabarti A, Shah A, et al. Allergic bronchopulmonary aspergillosis: review of literature and proposal of new diagnostic and classification criteria. Clin Exp Allergy 2013; 43: 850-873.

105 Wark PA, Hensley MJ, Saltos N, et al. Anti-inflammatory effect of itraconazole in stable allergic bronchopulmonary aspergillosis: a randomized controlled trial. J Allergy Clin Immunol 2003; 111: 952-957.

106 Stevens DA, Schwartz HJ, Lee JY, et al. A randomized trial of itraconazole in allergic bronchopulmonary aspergillosis. N Engl J Med 2000; 342: 756-762.

107 Agbetile J, Bourne M, Fairs A, et al. Effectiveness of voriconazole in the treatment of Aspergillus fumigatus-associated asthma (EVITA3 study). J Allergy Clin Immunol 2014; 134: 33-39.

$108 \mathrm{Li}$ JX, Fan LC, Li MH, et al. Beneficial effects of Omalizumab therapy in allergic bronchopulmonary aspergillosis: a synthesis review of published literature. Respir Med 2017; 122: 33-42. 
Voskamp AL, Gillman A, Symons K, et al. Clinical efficacy and immunologic effects of omalizumab in allergic bronchopulmonary aspergillosis. J Allergy Clin Immunol Pract 2015; 3: 192-199.

110 Dahlen SE, Malmstrom K, Nizankowska E, et al. Improvement of aspirin-intolerant asthma by montelukast, a leukotriene antagonist: a randomized, double-blind, placebo-controlled trial. Am J Respir Crit Care Med 2002; 165: 9-14.

111 Keith PK, Koch C, Djandji M, et al. Montelukast as add-on therapy with inhaled corticosteroids alone or inhaled corticosteroids and long-acting beta-2-agonists in the management of patients diagnosed with asthma and concurrent allergic rhinitis (the RADAR trial). Can Respir J 2009; 16: Suppl. A, 17A-31A.

112 Lazarus SC, Chinchilli VM, Rollings NJ, et al. Smoking affects response to inhaled corticosteroids or leukotriene receptor antagonists in asthma. Am J Respir Crit Care Med 2007; 175: 783-790.

113 Cameron EJ, Chaudhuri R, Mair F, et al. Randomised controlled trial of azithromycin in smokers with asthma. Eur Respir J 2013; 42: 1412-1415.

114 Gibeon D, Batuwita K, Osmond $\mathrm{M}$, et al. Obesity-associated severe asthma represents a distinct clinical phenotype: analysis of the British Thoracic Society Difficult Asthma Registry patient cohort according to BMI. Chest 2013; 143: 406-414.

115 Dias-Junior SA, Reis M, de Carvalho-Pinto RM, et al. Effects of weight loss on asthma control in obese patients with severe asthma. Eur Respir J 2014; 43: 1368-1377.

116 Pakhale S, Baron J, Dent R, et al. Effects of weight loss on airway responsiveness in obese adults with asthma: does weight loss lead to reversibility of asthma? Chest 2015; 147: 1582-1590.

117 van Huisstede A, Rudolphus A, Castro CM, et al. Effect of bariatric surgery on asthma control, lung function and bronchial and systemic inflammation in morbidly obese subjects with asthma. Thorax 2015; 70: 659-667.

118 Evans S, Kurukulaaratchy RJ. The effect of bariatric surgery in the difficult asthma-obesity phenotype: a case report. J Asthma 2013; 50: 52-55.

119 Toh JJ, Pasupathy S, Poopalalingam RA, et al. Can bariatric surgery be performed safely in patients with severe treatment-resistant asthma? Obes Surg 2014; 24: 334-336. 